\title{
Effect of Strain Rate on Compressive Behaviour of Silicone Rubber
}

\author{
Kohei Tateyama ${ }^{1, *}$, Hiroyuki Yamada $^{2}$, and Nagahisa Ogasawara $^{2}$ \\ ${ }^{1}$ Graduate Student, Graduate School of Science and Engineering, National Defense Academy, Kanagawa 239-8686, Japan \\ ${ }^{2}$ Department of Mechanical Engineering, National Defense Academy, Kanagawa 239-8686, Japan
}

\begin{abstract}
The purpose of this study is to evaluate the effect of strain rate on compressive behaviour of silicone rubber. The silicone rubber which was used as biomimetic material was prepared as a specimen. The shape of specimen was cubic and each side length was $10 \mathrm{~mm}$. In this study, a dynamic compressive test was performed using a drop-weight testing machine at the strain rate of approximately $10^{1} \mathrm{~s}^{-1}$, which can be detect the compressive stress for a long time without any disturbance. For comparison, a quasi-static compressive test was performed using the universal testing machine at the strain rate of $10^{-4}$ to $10^{-1} \mathrm{~s}^{-1}$. In the deformation process of the silicone rubber, the flow stress did not increase at the early stage of deformation. When the strain reached 0.6 , the flow stress was increased with increasing strain. This deformation process was considered to be due to rubber elasticity. It was confirmed that the silicone rubber showed an increase of the flow stress with increasing strain rate, which showed general behaviour of soft materials such as rubber. In the silicone rubber, the flow stress with respect to the strain rate could be simply expressed with the Cowper-Symonds constitutive equation.
\end{abstract}

\section{Introduction}

Helmets and protectors are often used to protect the human body from impact loading such as collision of flying objects. Therefore, it is important to accurately evaluate its protective performance. In the previous study, the protective performance has been evaluated by the degree of dent at the clay [1]. However, when an impact load is applied to the human body protected by the protector, the muscle and the visceral tissues are sometimes damaged by the propagation of stress waves or shock waves, even if the visible scars do not appear [2]. Such damages are called "blunt trauma" and it is difficult to evaluate by the previous testing methods using clay. Therefore, a method that also can evaluate the blunt trauma is required.

In recent years, the damage assessment using human dummies made of soft materials such as silicone rubber has been conducted [3]. In this method, since it is possible to obtain the pressure-history and the displacement-history generated in a human dummy, it is expected as a method for evaluating the blunt trauma. In order to evaluate precisely protection performance using human dummies, it is necessary to know the mechanical properties of silicone rubber. However, there are few reports on the dynamic properties of soft materials such as the silicone rubber, because these have the characteristic of large deformation [4].

Thus, the purpose of this study is to evaluate the effect of strain rate on compressive behaviour of silicone rubber. We performed the quasi-static and the dynamic compressive tests on the silicone rubber which was a biomimetic material.

\section{Experimental Procedures}

\subsection{Material}

In this study, the silicone rubber which was used as biomimetic material was prepared as a specimen. When the specimen was produced by die-casting with a metal mold, there was a problem that the shape of specimen collapsed at the time of peeling from this mold. Therefore, the specimen cut out from a large-sized block sample $(200 \times 200 \times 300 \mathrm{~mm})$ was used as the specimen. In the cutting of specimen, the cutting surface was curved with a thin blade such as a razor. Therefore, a thick blade like a kitchen knife was used for cut out the specimen.

Figure 1 shows the images of silicone rubber specimen. The shape of specimen was cubic and each side length was $10 \mathrm{~mm}$.

\subsection{Definition of initial thickness}

Since the silicone rubber deforms with a very weak force, the deformation occurs at the time of measuring the initial thickness. Therefore, in the present study, the thickness measured with a dial gauge which mounted a planar measuring probe with a diameter of $15 \mathrm{~mm}$ was 


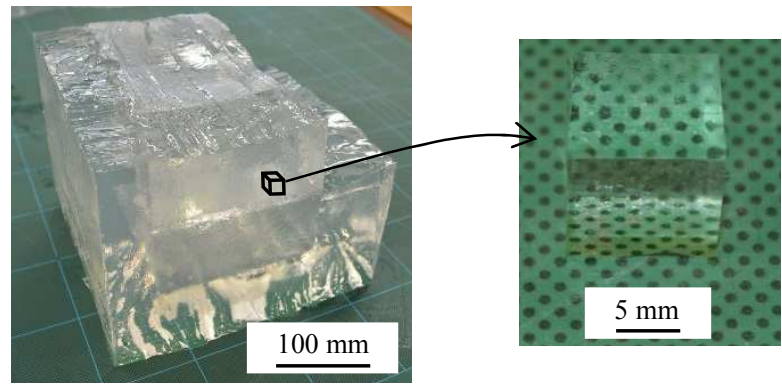

Fig. 1. Images of silicone rubber specimen.

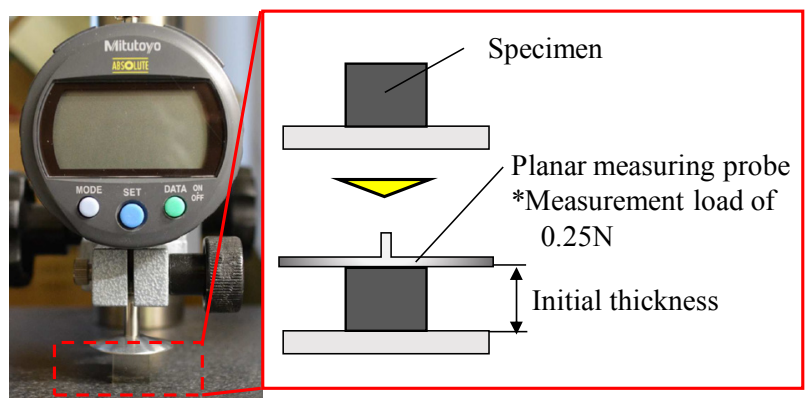

Fig. 2. Measurement of thickness at the silicone rubber specimens using the dialgage which mounted a planar measuring probe.

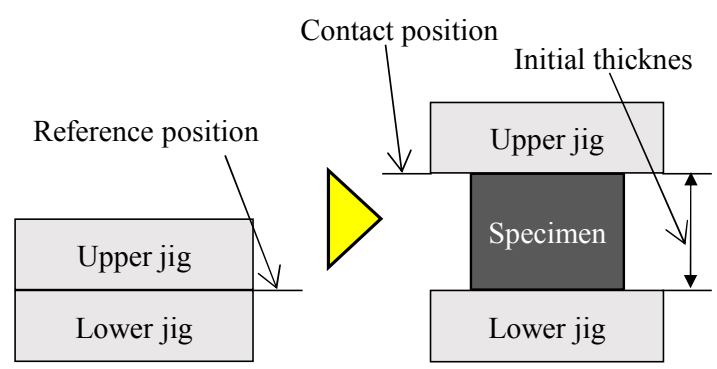

Fig. 3. The conceptual diagram of the contact position between specimen and jig.

defined as the initial thickness. Figure 2 shows the measurement of thickness at the silicone rubber specimens.

The mass of the planar measuring probe is $2.55 \times 10^{-3}$ $\mathrm{kg}$. Thus, the measuring force is approximately $0.25 \mathrm{~N}$. By measuring the thickness of specimen with this small constant load, the measurement error due to measuring force was reduced.

\subsection{Quasi-static compressive test}

The quasi-static compressive test was conducted using the universal testing machine (5500R, Instron).

The contact position between specimen and jig was determined by using the initial thickness which was defined in the previous section. Figure 3 showed the conceptual diagram of the contact position between specimen and jig. First, the position where upper and

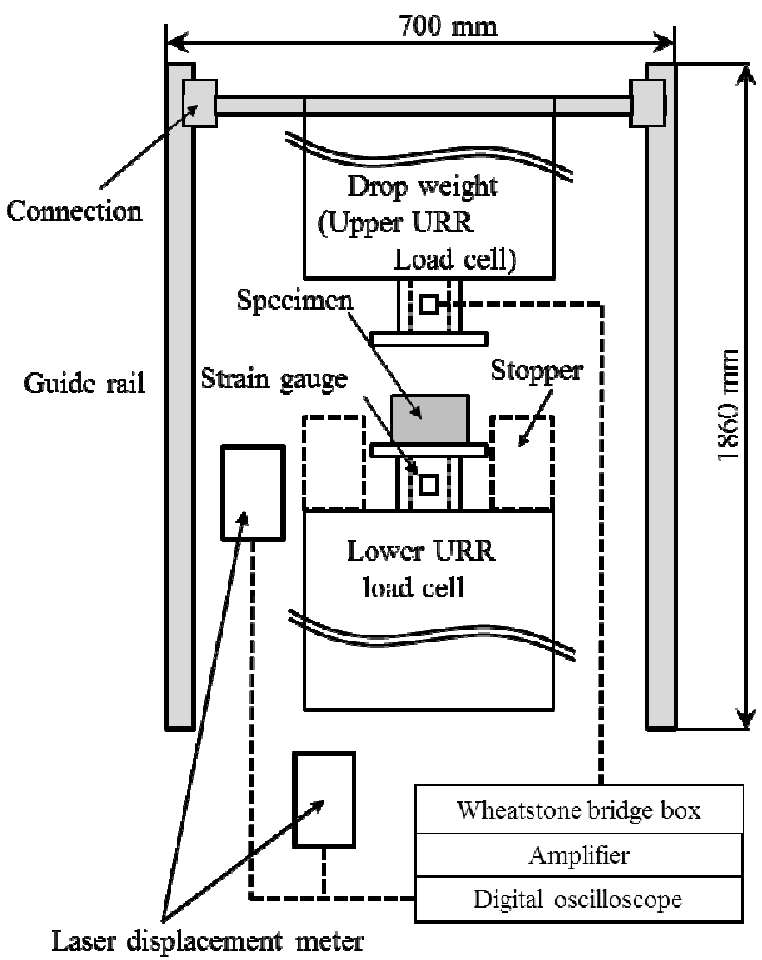

Fig. 4. The conceptual diagram of the contact position between specimen and jig [4].

lower jigs contact each other without specimen is taken as the reference position. At this time, the pressure distribution between the jigs was observed using a pressure measurement film (Fuji Film, PRESCALE-LW, measured pressure band 2.5 to $10 \mathrm{MPa}$ ), and the pressure distribution between the jigs was adjusted to be even. After setting specimens, the gap between upper and lower jigs was set to the initial thickness of each specimen by the control of machine, and this position was defined as the contact point and it was regarded as strain zero.

The quasi-static compressive test was carried out at the initial strain rates of approximately $1.7 \times 10^{-3}, 1.7 \times 10^{-2}$ and $1.7 \times 10^{-1} \mathrm{~s}^{-1}$. These tests were conducted three times for each strain rate.

\subsection{Dynamic compressive test}

The dynamic compressive test at the strain rate of approximately $10^{1} \mathrm{~s}^{-1}$ was conducted using a drop-weight testing machine. [4]. This machine was used a universal rate range (URR) load cell in order to reduce the influence of the reflected stress waves [5]. URR load cell can detect the compressive stress for a long time without any disturbance using reduction technique of reflection stress wave. Therefore, it is suitable to evaluate the large deformable materials such as silicone rubber [5].

This testing machine was consisted of a drop-weight, guide rail and URR load cells. Figure 4 shows the schematic view of a drop-weight testing machine [4]. The recording system of this testing machine was comprised of a Wheatstone bridge box and differential amplifier (5305, NF Co., Ltd.), and digital oscilloscope (DL750, Yokogawa Meters \& Instruments Co., Ltd.). The 
displacement of the drop-weight was measured using a laser displacement meter (LK-G5000V, KEYENCE Co., Ltd. sampling rate: $100 \mathrm{k} \mathrm{Hz}$ ).

In this testing machine, it is possible to measure the stress equilibrium of a specimen by using upper and lower load cells. It was confirmed that stress equilibrium was achieved under this experimental condition.

The dynamic compressive test using the drop-weight testing machine was carried out at approximately $5.4 \times$ $10^{1} \mathrm{~s}^{-1}$. As with the quasi-static test, the contact position was determined using the initial thickness which was defined in the previous section.

\section{Results and Discussion}

\subsection{Stress-strain relationship}

Figure 5 shows the typical stress-strain relationship obtained from the quasi-static and dynamic compressive test of silicone rubber. The oscillation in the dynamic test result is the superimposition of the white noise from the power supply. In the deformation process of the silicone rubber, the rate of increase in flow stress was very low at the early stage of deformation. When the strain reached approximately 0.6 , the flow stress was increased with increasing strain. This deformation process was considered to be due to rubber elasticity, which occurs when the network structure of the polymer chain at room temperature is in the rubber state [6].

In addition, it was confirmed that the silicone rubber showed an increase of the flow stress with increasing strain rate, which showed general behaviour of soft materials such as rubber.

\subsection{Effect of strain rate}

In order to investigate the effect of strain rate on the compressive stress of the silicone rubber in detail, the variation of that compressive stress was plotted as a function of strain rate using a double logarithm at each strain $(\varepsilon=0.3-0.5)$, as shown in Figure 6. It has been reported that general polymeric materials have the high rate of increase of the flow stress at the dynamic and impact strain rates compared with the quasi-static strain rate [7]. In the silicone rubber used in this study, the same tendency as the previous reports was observed.

From this result, we consider a constitutive equation. The flow stress with respect to the strain rate could be expressed with exponential function defined by following equations.

$$
\sigma=\sigma_{0}\left[1+\left(\frac{\dot{\varepsilon}}{\gamma}\right)^{m}\right]
$$

where $\sigma_{0}$ is the static stress, $m$ and $\gamma$ are the strain rate parameters. The above equation is called the CowperSymonds constitutive equation [8]. This constitutive equation is widely used to represent the strain rate sensitivity of metal materials in a simple way.

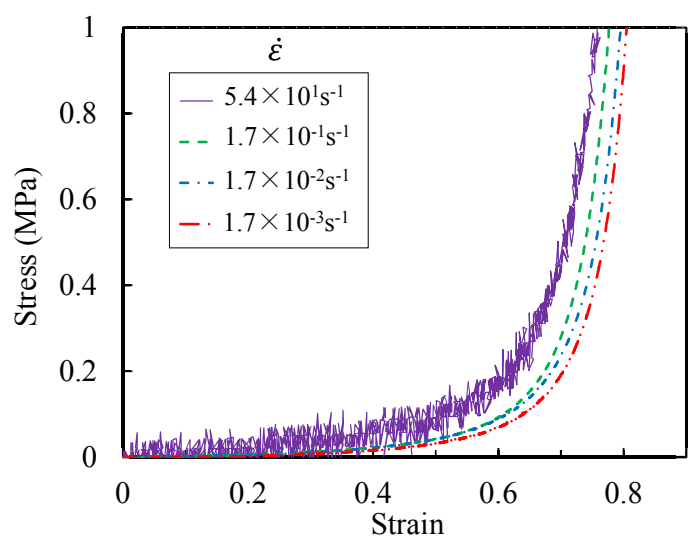

Fig. 5. The typical stress-strain relationship obtained from the quasi-static and dynamic compressive test of silicone rubber.

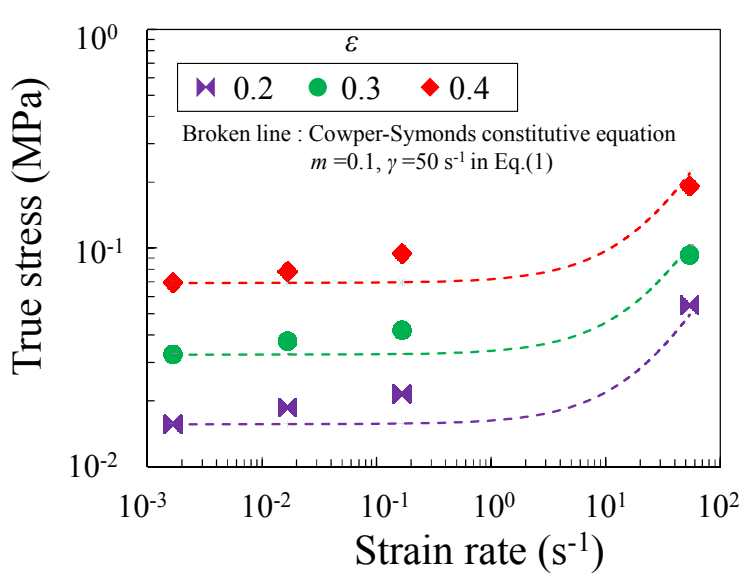

Fig. 6. The variation in compressive stress plotted as a function of strain rate using a double logarithm at each strain.

The broken line indicate in Figure 6 shows the result obtained from the Cowper-Symonds constitutive equation. Where, the value of $m$ is 0.1 and $\gamma$ is $50 \mathrm{~s}^{-1}$. In the present study, the value of $\sigma_{0}$ was substituted for the stress at the strain rate of $1.7 \times 10^{-3} \mathrm{~s}^{-1}$.

In the silicone rubber, the flow stress with respect to the strain rate could be simply expressed with the Cowper-Symonds constitutive equation. However, in the quasi-static strain rate range from $1.7 \times 10^{-2}$ to $1.7 \times 10^{-1} \mathrm{~s}^{-1}$, the value obtained from the Cowper-Symonds constitutive equation did not match the experimental results. Therefore, in order to express the stress-strain relation more accurately, it is necessary to consider the others constitutive equation.

\section{Summary}

In this study, the quasi-static and the dynamic compressive tests were performed on the silicone rubber which was a biomimetic material, and the effect of strain rate was investigated. The results can be summarized as follows.

(1) In the deformation process of the silicone rubber, the rate of increase in flow stress was very low at the early stage of deformation. This deformation process was considered to be due to rubber elasticity which 
occurs when the network structure of the polymer chain at room temperature is in the rubber state.

(2) It was confirmed that the silicone rubber showed an increase of the flow stress with increasing strain rate.

(3) In the silicone rubber, the flow stress with respect to the strain rate could be simply expressed with the Cowper-Symonds constitutive equation. However, in the quasi-static strain rate range from $1.7 \times 10^{-2}$ to $1.7 \times 10^{-1} \mathrm{~s}^{-1}$, the value obtained from the CowperSymonds constitutive equation did not match the experimental result.

The authors are deeply grateful to Dr. Sakamoto, Mr. Tsukada (the Acquisition, Technology \& Logistics Agency) and Mr. Ishihara (undergraduate student at the National Defense Academy), for their assistance.

\section{References}

1. M. B. Mukasey, J. L. Sedgwick, D. W. Hagy, US Department of Justice (2005)
2. A. Ramasamy, A. M. Hill, S. Masouros, I. Gibb, A. M. Bull, J. C. Clasper, J. Roy. Soc. Interface, 8, 58 (2011)

3. K. Morimoto, $R \& D$ review of Toyota $C R D L, 27,1$ (1992)

4. K. Tateyama, H. Yamada, N. Ogasawara, Polym. Test., 52 (2016)

5. K. Tateyama, C. Kakuno, N. Ogasawara, H. Yamada, J. JSEM, 17, 131 (2017)

6. K. Tashiro, Japanese Journal of Polymer Science and Technology, 49, 9 (1992)

7. T. Adachi, S. Yamamura, M. Arai, H. Matsumoto, Trans. Jpn. Soc. Mech. Eng., A 65, 635 (1997)

8. G.R. Cowper, P.S. Symonds, Brown Univ., Applied Mathematics Report (1958) 